COMMENT. The Mozart effect was first observed in college students who showed induction of right frontal and left hemisphere EEG activity and enhancement of visual-spatial-temporal reasoning during and after listening to 10 minutes of the Mozart sonata tape. (Rauscher FH et al. Nature 1993; Sarnthein J et al. Neurol Res 1998). Relaxation tapes and minimalist music had no effect. These investigators also reported long-term enhancement of preschool children's spatial temporal reasoning during piano keyboard lessons but not computer lessons. Listening to music is also recommended as adjunct therapy in children with ADHD and adults with Parkinsonism or Alzheimer's disease. The Mozart effect in patients with epilepsy is explained by resonance of the cerebral cortex with the architecture of Mozart's music (Hughes JR et al. Clin Electroencephalogr 1998;29(3):109-119).

The variation in the Mozart response with cerebral localization of the epileptiform discharge may be a manifestation of the functional independence of sub-components of musical expression. The left hemisphere is dominant for rhythm, tune recognition, and pitch perception, while the right hemisphere is involved in timbre or quality of tone perception. The differentiation of pitch requires not only auditory, but also, visual interpretation and mental imagery. Listening to Mozart enhances cortical cerebral activity used in spatial-temporal reasoning (Platel, Frackowiak et al. Brain 1997;120:229-243). String music (string Mozart 448) with a larger number of higher harmonics than piano Mozart 448 had no effect on epileptiform discharges. (Lin L-C et al. Epilepsy Res 2010;89(2-3):238-245).

\title{
ATYPICAL PRESENTATIONS/COMORBIDITIES OF BCECTS
}

The frequency of atypical features among 196 patients (118 male and 78 female; mean age at diagnosis 7.64 years, range 1.5-14) with benign childhood epilepsy with centrotemporal spikes (BCECTS) was determined by a retrospective chart study in four pediatric neurology outpatient clinics in Israel between the years 1991 and 2008. Mean duration of follow-up was 4.43 years (range 2-11). Comorbidities or presentations included electrical status epilepticus in slow waves sleep (ESES) in 9 (4.6\%) patients, Landau-Kleffner syndrome in $4(2 \%)$, BCECTS with frequent refractory seizures in 3 $(1.5 \%)$, BCECTS with falls in $2(1 \%)$, atypical variant in $1(0.5 \%)$, and oromotor dysfunction in $1(0.5 \%)$. Sixty-one $(31 \%)$ had ADHD, $43(21.9 \%)$ had specific cognitive deficits, and $23(11.7 \%)$ had behavioral disorders, including aggressiveness, anxiety, depression, and pervasive developmental disorder. (Tovia E, Goldberg-Stern H, Zeev $\mathrm{BB}$, et al. The prevalence of atypical presentations and comorbidities of benign childhood epilepsy with centrotemporal spikes. Epilepsia Sept 2011;52(8):1483-1488). (Respond: Uri Kramer MD, Pediatric Neurology Unit, Tel Aviv, Sourasky, Medical Center, 6 Weitzman St, Tel Aviv 65211, Israel. E-mail: umkramer@netvision.net.il).

COMMENT. BCECTS is the most prevalent of benign focal epilepsies of childhood, accounting for $15-25 \%$ of epilepsy syndromes in children $<15$ years of age. Age at onset ranges from 3-13 years, with peak incidence at 7-8 years. The male-tofemale ratio is $3: 2$. Hemifacial motor seizures are preceded by drooling and oromotor apraxia (Lombroso, 1967; Roulet, Deonna et al, 1989, 1993), and frequently followed by ipsilateral hand and leg involvement, usually nocturnal. Apart from ESES, an EEG 
diagnosis manifesting S/W activity for $85 \%$ of slow-wave sleep time, and found in $5 \%$ of the above series, atypical forms of BCECTS were uncommon. In contrast, comorbidities were frequent, and especially, ADHD, specific cognitive deficits, and behavior disorders.

\section{HEADACHE DISORDERS}

\section{HEADACHE AND IDIOPATHIC EPILEPSY}

Clinical characteristics of headache in 1,264 children with newly diagnosed idiopathic epilepsy were studied from 2006 to 2009 in five Italian Child Neurology University Centers. Seizures were generalized in 61 (tonic-clonic in 45) and partial in 69 patients (benign rolandic in 15). Of 130 children who showed an associated peri-ictal and/or interictal headache, $62 \%$ had post-ictal headache, $30 \%$ pre-ictal, and $57.6 \%$ interictal headache. Rare cases of ictal epileptic headache ("migralepsy") were excluded. Preictal, post-ictal, and inter-ictal headaches met criteria for migraine in $93 \%, 81.4 \%$ and $87 \%$, respectively. Migraine without aura was most commonly associated with partial epilepsy, and occurred in $82 \%$ patients with peri-ictal headache and in $76.5 \%$ patients with post-ictal headache. Tension-type headache occurred in $19 \%$ patients. Focal epileptiform discharges in 39 children with partial epilepsy were frontal in $31 \%$, temporal in $13 \%$, and occipital in $41 \%$. Peri-ictal headaches were post-ictal in 29 (74\%) and preictal in $10(26 \%)$ patients with focal EEGs. Type of headache was not significantly correlated with localization of EEG abnormalities. (Verrotti A, Coppola G, Spalice A, et al. Peri-ictal and inter-ictal headache in children and adolescents with idiopathic epilepsy: a multicenter cross-sectional study. Childs Nerv Syst September 2011;27:1419-1423). (Address: Dr A Verrrotti, Department of Pediatrics, University of Chieti, Italy).

COMMENT. This study confirms the prevalence of post-ictal headache associated with idiopathic epilepsy and an association of partial epilepsy with peri-ictal headache, most commonly migrainous. EEG is not routinely indicated in the evaluation of children with headache but may be appropriate in patients with atypical migrainous aura or episodic loss of consciousness. Several studies have addressed the utility of the EEG in children with headache, some concluding that a beneficial effect of anticonvulsant drugs in treatment of chronic migraine is not correlated with EEG epileptiform abnormalities. In 30 children with migraine studied in the 1970s and treated with the anticonvulsant, phenytoin, $77 \%$ had a decreased recurrence of headaches. The response rate was $61 \%$ in 13 patients with abnormal EEGs and $88 \%$ in 17 with normal EEGs, and the difference was not significant. Response to AED was independent of the EEG abnormality (Millichap JG. Child's Brain 1978;4:95-104). In an analysis of records of 257 children with chronic headache, the EEG showed epileptiform activity in $12 \%$ and slowing in 8\%. (Kramer U et al. Brain Dev 1994;16:304-308).

The Cochrane Central Register of Controlled Trials of anticonvulsants in the prophylaxis of migraine in adults recorded 14 reported trials comparing AED with placebo in a total of 2024 patients: 4 trials with divalproex sodium, 2 sodium valproate, 3 topiramate, 2 gabapentin, and one trial each of carbamazepine, clonazepam, and lamotrigine. Sodium valproate/divalproex sodium and topiramate are effective in reducing migraine frequency and are reasonably well tolerated. Neither clonazepam nor 